\title{
MODULUS OF CONTINUITY OF OPERATOR FUNCTIONS
}

\author{
YU. B. FARFOROVSKAYA AND L. NIKOLSKAYA
}

\begin{abstract}
Let $A$ and $B$ be bounded selfadjoint operators on a separable Hilbert space, and let $f$ be a continuous function defined on an interval $[a, b]$ containing the spectra of $A$ and $B$. If $\omega_{f}$ denotes the modulus of continuity of $f$, then

$$
\|f(A)-f(B)\| \leq 4\left[\log \left(\frac{b-a}{\|A-B\|}+1\right)+1\right]^{2} \cdot \omega_{f}(\|A-B\|) .
$$

A similar result is true for unbounded selfadjoint operators, under some natural assumptions on the growth of $f$.
\end{abstract}

\section{INTRODUCTION}

If $A$ is a selfadjoint operator on a Hilbert space, the spectral theorem makes it possible to define $f(A)$ for any Borel function $f$ on the real line $\mathbb{R}$. In this paper we are going to study the continuity properties of the map $A \mapsto f(A)$.

Definition (see [1]). A continuous function $f$ on $\mathbb{R}$ is said to be operator continuous if $\lim _{n \rightarrow \infty}\left\|f\left(A+K_{n}\right)-f(A)\right\|=0$ whenever $\lim _{n \rightarrow \infty}\left\|K_{n}\right\|=0$ for any selfadjoint operator $A$ and any bounded selfadjoint operators $K_{n}$ on a separable Hilbert space $H$.

In 12, Farforovskaya gave an example implying that the function defined on the real line by $x \mapsto|x|$ is not operator continuous (see pages 169-172). It can be shown that, even infinitely smoothed at zero, this function will still not be operator continuous. On the other hand, the results of Peller show that the functions belonging to the Besov class $B_{\infty 1}^{1}$ are operator continuous (see, e.g., [2]). Moreover, for any selfadjoint operator $A$ and any function $f$, there exists a constant $C>0$ such that the following linear estimate is fulfilled:

$$
\|f(A+K)-f(A)\| \leq C\|K\| .
$$

Earlier, Birman and Solomyak obtained estimate (1) for any bounded selfadjoint operator and the functions $f$ such that $f^{\prime} \in \operatorname{Lip}(\alpha), 0<\alpha \leq 1$ (see 3 ). In the case of normal operators $A$ and $B$ and a function $f$ of class $\operatorname{Lip}(\alpha)$, the difference $f(A)-f(B)$ was estimated in [14] and [15].

In this paper we discuss the case of highly nonsmooth functions; we use the modulus of continuity as a way to "measure" the continuity of the corresponding operator function. Theorem 10 estimates the norm $\|f(A)-f(B)\|$ for any continuous function on an interval containing the spectra of the two bounded selfadjoint operators $A$ and $B$. Many of the earlier results can be obtained as corollaries to this theorem. In particular, the inequality proved in Theorem 10 produces an estimate of the norm $\|f(A)-f(B)\|$ in the case of $\operatorname{Lip}(\alpha)$-functions.

Theorem 15 treats the case of unbounded selfadjoint operators. The estimate obtained in that theorem yields a sufficient condition of operator continuity. The assumptions on

2000 Mathematics Subject Classification. Primary 47B15.

Key words and phrases. Selfadjoint operator, operator function, modulus of continuity. 
the function are not too restrictive. In a natural way, they control the growth of the function at infinity, but not its smoothness. For instance, this theorem shows that the function on the real line defined by $x \mapsto \frac{|x|}{\log ^{3}|x|}$ is operator continuous.

This paper is organized as follows. In $\S 1$, we prove some lemmas about HadamardSchur multipliers. The proofs of Theorems 10 and 15 are contained in $\S 2$ and $\S 3$, respectively.

\section{§1. Lemmas on some SPECIAL HadAmard-Schur Multipliers}

Recall that the Hadamard product of two matrices $M=\left(m_{i j}\right)$ and $A=\left(a_{i j}\right)$ is defined as $M \circ A=\left(m_{i j} a_{i j}\right)$. A matrix $M$ is a Hadamard-Schur multiplier if

$$
\|M\|_{\mathcal{H}}=\sup \left\{\|M \circ A\| \mid A: l^{2} \longrightarrow l^{2},\|A\| \leq 1\right\}<\infty \text {. }
$$

We need the following remarkable theorem due to G. Bennett [4] (see also [5] for generalizations).

Theorem 0. 1. A Toeplitz matrix $M=\left(m_{i j}\right), m_{i j}=c_{i-j}, i, j \in \mathbb{N}$, is a HadamardSchur multiplier if and only if there exists a (bounded, complex Borel) measure $\mu$ on the circle $\mathbb{T}$ with $\widehat{\mu}(m)=c_{m}, m \in \mathbb{Z}$. Moreover, we then have $\|M\|_{\mathcal{H}}=\|\mu\|$.

2. A Hankel matrix $M=\left(m_{i j}\right), m_{i j}=c_{i+j}, i, j \in \mathbb{N}$, is a Hadamard-Schur multiplier if there exists a measure $\mu$ on the circle with $\widehat{\mu}(m)=c_{m}, m \in \mathbb{N}$, and then $\|M\|_{\mathcal{H}} \leq\|\mu\|$.

The next lemma was proved in [5].

Lemma 1. For a function $\varphi: \mathbb{N} \longrightarrow \mathbb{R}$, let $M_{n, \varphi}$ be the $(n \times n)$-matrix with the entries

$$
m_{i j}= \begin{cases}1 & \text { if } i \leq \varphi(j), \quad 0 \leq j<n \\ 0 & \text { if } i>\varphi(j), \quad 0 \leq j<n\end{cases}
$$

Put $N(\varphi, n)=\operatorname{card}\{[\varphi(j)]: 0 \leq j<n\}$, where [.] stands for the integral part of a number. Then

$$
c \cdot \log N(\varphi, n) \leq\left\|M_{n, \varphi}\right\|_{\mathcal{H}} \leq \log N(\varphi, n)+1
$$

where $c>0$ is an absolute constant.

Remark. The classical Matsaev theorem [6] on triangular truncation is a particular case of this lemma corresponding to $\varphi(j)=j$.

In the following lemmas we calculate or estimate the norms of some specific multipliers.

Let $k>0$ be a positive number, and let $T_{k}=\left(m_{i j}\right)$ denote the following infinite symmetric Toeplitz matrix:

$$
T_{k}=\left(\begin{array}{cccc}
\frac{1}{k} & \frac{1}{k+1} & \frac{1}{k+2} & \ldots \\
\frac{1}{k+1} & \frac{1}{k} & \frac{1}{k+1} & \ldots \\
\frac{1}{k+2} & \frac{1}{k+1} & \frac{1}{k} & \ldots \\
\ldots & \ldots & \ldots & \ldots
\end{array}\right) .
$$

In other words,

$$
m_{i j}=\frac{1}{|i-j|+k}=c_{i-j}, \quad c_{m}=\frac{1}{k+|m|}, \quad m \in \mathbb{Z} .
$$

Lemma 2. $\left\|T_{k}\right\|_{\mathcal{H}}=\frac{1}{k}$.

Proof. Let $f_{k}$ be the function defined on the real line $\mathbb{R}$ by the formula

$$
f_{k}(x)=\frac{1}{k+|x|}, \quad x \in \mathbb{R} .
$$


We have

$$
f_{k}(m)=\frac{1}{k+|m|}=c_{m}, \quad m \in \mathbb{Z}
$$

The function $f_{k}$ is positive, continuous, and even. Moreover, it is convex and monotone decreasing on $\mathbb{R}_{+}$. Then, by a theorem of Polya [7, $f_{k}$ is the Fourier transform of a positive measure on $\mathbb{R}$. (By the way, it is easy to verify this directly.) The Bochner theorem (see [8]) shows that $f_{k}$ is a positive definite function. This means that the inequality

$$
\sum_{i, j=1}^{N} c_{i} \overline{c_{j}} f_{k}\left(x_{i}-x_{j}\right) \geq 0
$$

is true for every choice of $x_{1}, \ldots, x_{N}$ on the real line and every choice of complex numbers $c_{1}, \ldots, c_{N}$.

In particular, for arbitrary complex numbers $c_{1}, \ldots, c_{N}$ we have

$$
\sum_{i, j=1}^{N} c_{i} \overline{c_{j}} f_{k}(i-j) \geq 0 .
$$

By Herglotz's theorem [9], it follows that the measure $\mu_{k}$ on $\mathbb{T}$ whose Fourier coefficients are equal to $f_{k}(m), m \in \mathbb{Z}$, is positive. So,

$$
\left\|\mu_{k}\right\|=\mu_{k}(\mathbb{T})=\widehat{\mu_{k}}(0)=f_{k}(0)=\frac{1}{k} .
$$

By Theorem $0,\left\|T_{k}\right\|_{\mathcal{H}}=\frac{1}{k}$. The lemma is proved.

Now we fix two integers $k$ and $n$ with $k<n$ and denote by $K_{n}=\left(m_{i j}\right)_{1 \leq i, j \leq n}$ the following finite Toeplitz matrix:

$$
K_{n}=\left(\begin{array}{ccccccc}
0 & \ldots & 0 & \frac{1}{k} & \frac{1}{k+1} & \ldots & \frac{1}{n-1} \\
0 & \ldots & 0 & 0 & \frac{1}{k} & \ldots & \frac{1}{n-2} \\
\ldots & \ldots & \ldots & \ldots & \ldots & \ldots & \ldots \\
0 & 0 & 0 & 0 & 0 & \ldots & \frac{1}{k} \\
0 & 0 & 0 & 0 & 0 & \ldots & 0 \\
\ldots & \ldots & \ldots & \ldots & \ldots & \ldots & \ldots \\
0 & 0 & 0 & 0 & 0 & \ldots & 0
\end{array}\right),
$$

i.e., $m_{i j}=\frac{1}{j-i}$ if $i+k \leq j \leq n$ and $m_{i j}=0$ otherwise.

\section{Lemma 3.}

$$
\left\|K_{n}\right\|_{\mathcal{H}} \leq \frac{1}{k} \cdot[\log (n-k)+1] .
$$

Proof. This follows from Lemmas 1 and 2 and the fact that if $M=M_{1} \circ M_{2}$, then $\|M\|_{\mathcal{H}} \leq\left\|M_{1}\right\|_{\mathcal{H}}\left\|M_{2}\right\|_{\mathcal{H}}$.

Remark. If we extend the matrix $K_{n}$ up to the infinite Toeplitz matrix

$$
K_{\Delta}=\left(m_{i j}\right)_{i, j \geq 1}, \quad m_{i j}= \begin{cases}\frac{1}{j-i} & \text { if } j \geq i+k, \\ 0 & \text { otherwise, }\end{cases}
$$

then the matrix $K_{\Delta}$ is still a multiplier with $\left\|K_{\Delta}\right\|_{\mathcal{H}} \leq \frac{1}{\sqrt{k}}$. A simple way to see this is to employ the classical inequality of Schur [10]. Namely, if $M=\left(m_{i j}\right)_{i, j \geq 1}$ and $r(M)=\sup _{i \geq 1}\left\|M e_{i}\right\|$, where the $e_{i}, i \geq 1$, are the basis vectors of $l^{2}$, then

$$
\|M\|_{\mathcal{H}} \leq \min \left(r(M), r\left(M^{*}\right)\right) .
$$


Therefore, estimate (2) may look not quite satisfactory, because of the growing factor that depends on the size of the matrix. On the other hand, estimation of the norm of the multiplier $K_{\Delta}$ by $\frac{1}{\sqrt{k}}$ is too rough for our purposes. Relative to the parameter $k$, we need to reduce the norm of the multiplier $K_{n}$ to $\frac{1}{k}$ precisely. But it is easily seen that no estimate of the form $\left\|K_{\Delta}\right\|_{\mathcal{H}} \leq \frac{c}{k}$ is possible. Indeed, the Toeplitz multiplier $K_{\Delta}$ is generated by the measure $\nu_{k}=\sum_{n \geq 0} \frac{z^{n}}{k+n}, z \in \mathbb{T}$. However, by Theorem 0 ,

$$
\left\|K_{\Delta}\right\|_{\mathcal{H}}=\int_{\mathbb{T}}\left|\sum_{n \geq 0} \frac{z^{n}}{k+n}\right| d m(z)=\frac{1}{k} \int_{\mathbb{T}}\left|\sum_{n \geq 0} \frac{z^{n}}{1+\frac{n}{k}}\right| d m(z) .
$$

Suppose there exists an absolute constant $c$ such that

$$
\int_{\mathbb{T}}\left|\sum_{n \geq 0} \frac{z^{n}}{1+\frac{n}{k}}\right| d m(z) \leq c .
$$

Since in the space of measures the sphere is weakly compact, it follows that there exists a measure $\mu$ on the circle whose Fourier coefficients are $\widehat{\mu}(n)=1, n \geq 0 ; \widehat{\mu}(n)=0, n<0$.

The latter is impossible because of the theorem of H. Helson (see [11]) saying that a sequence of zeros and ones is a sequence of Fourier coefficients for a measure $\mu$ if and only if it coincides with a periodic sequence except for finitely many terms.

Thus, the presence of a growing factor in estimate (2) is indispensable.

For two fixed integers $k$ and $n$ with $k<n$, we denote by $F_{k, n}=\left(m_{i j}\right)_{i, j \geq 1}$ the following infinite matrix:

$$
\begin{aligned}
F_{n, k}= & \left(\begin{array}{ccccc}
\frac{1}{n} & \frac{1}{n+1} & \frac{1}{n+2} & \ldots & \ldots \\
\frac{1}{n-1} & \frac{1}{n} & \frac{1}{n+1} & \ldots & \ldots \\
\ldots & \ldots & \ldots & \ldots & \ldots \\
\frac{1}{k} & \frac{1}{k+1} & \frac{1}{k+2} & \ldots & \ldots \\
0 & 0 & 0 & 0 & \ldots \\
\ldots & \ldots & \ldots & \ldots & \ldots
\end{array}\right), \\
m_{i j}= & \left\{\begin{array}{cccc}
\frac{1}{j-i+n} & \text { if } i=1,2, \ldots, n-k+1, j \geq 1, \\
0 & \text { if } i>n-k+1 .
\end{array}\right.
\end{aligned}
$$

Lemma 4. $\left\|F_{k, n}\right\|_{\mathcal{H}} \leq \frac{1}{k}$.

Proof. Let $J_{p}=\left(m_{i j}\right)_{i, j \geq 1}, p \in \mathbb{N}$, be the symplectic matrix

$$
\begin{aligned}
& J_{p}=\left(\begin{array}{ccccccc}
0 & \ldots & 0 & 1 & 0 & 0 & \ldots \\
0 & \ldots & 1 & 0 & 0 & 0 & \ldots \\
\ldots & \ldots & \ldots & \ldots & \ldots & \ldots & \ldots \\
1 & 0 & 0 & \ldots & \ldots & \ldots & \ldots \\
0 & 0 & 0 & 0 & \ldots & \ldots & \ldots \\
\ldots & \ldots & \ldots & \ldots & \ldots & \ldots & \ldots
\end{array}\right), \\
& m_{i j}= \begin{cases}1 & \text { if } j=p+1-i, \quad 1 \leq i \leq p, \\
0 & \text { otherwise. }\end{cases}
\end{aligned}
$$

It is easily seen that for every $p \in \mathbb{N}$ and every matrix $M$ we have $\left\|J_{p} M\right\|_{\mathcal{H}} \leq\|M\|_{\mathcal{H}}$. Indeed, $\left\|J_{p}\right\|=1$ and $J_{p}(M \circ A)=\left(J_{p} M\right) \circ\left(J_{p} A\right)$ for every matrix $A$ and $p \in \mathbb{N}$. Therefore,

$$
\begin{aligned}
\left\|J_{p} M\right\|_{\mathcal{H}} & =\sup _{\|A\| \leq 1}\left\|\left(J_{p} M\right) \circ A\right\|=\sup _{\|A\| \leq 1}\left\|\left(J_{p} M\right) \circ\left(J_{p} A\right)\right\| \\
& =\sup _{\|A\| \leq 1}\left\|J_{p}(M \circ A)\right\| \leq \sup _{\|A\| \leq 1}\|M \circ A\|=\|M\|_{\mathcal{H}} .
\end{aligned}
$$


Now, let $\Gamma_{k}=\left(m_{i j}\right)_{i, j \geq 0}$ be the Hankel matrix

$$
\Gamma_{k}=\left(\begin{array}{cccc}
\frac{1}{k} & \frac{1}{k+1} & \frac{1}{k+2} & \ldots \\
\frac{1}{k+1} & \frac{1}{k+2} & \frac{1}{k+3} & \ldots \\
\frac{1}{k+2} & \frac{1}{k+3} & \frac{1}{k+4} & \ldots \\
\cdots & \ldots & \ldots & \ldots
\end{array}\right), \quad m_{i j}=\frac{1}{i+j+k}, \quad i, j \geq 0 .
$$

With $\Gamma_{k}$ we associate the measure $\mu$ on the circle with Fourier coefficients $\widehat{\mu}_{k}(m)=\frac{1}{k+|m|}$, $m \in \mathbb{Z}$. By Theorem 0 , we have $\left\|\Gamma_{k}\right\|_{\mathcal{H}} \leq \frac{1}{k}$. On the other hand, it is easy to show that $F_{n, k}=J_{n-k+1} \Gamma_{k}$. Thus, $\left\|F_{n, k}\right\|_{\mathcal{H}} \leq\left\|\Gamma_{k}\right\|_{\mathcal{H}} \leq \frac{1}{k}$. The lemma is proved.

Remark. It is possible to prove (of course, by different techniques) that if $k>0$ and $\left(c_{i}\right)_{i \geq 1},\left(d_{j}\right)_{j \geq 1}$ are two nonnegative sequences, then for the matrix $M=\left(m_{i j}\right)_{i, j \geq 1}$ with $m_{i j}=\frac{1}{k+c_{i}+d_{j}}$ we have $\|M\|_{\mathcal{H}} \leq \frac{1}{k}$.

We shall also need the following evident assertion.

Lemma 5. Let $\left(a_{i}\right)_{i \geq 1}$ be a bounded sequence, and let $M=\left(m_{i j}\right)_{i, j \geq 1}, m_{i j}=a_{i}$. Then $\|M\|_{\mathcal{H}} \leq \sup _{i}\left|a_{i}\right|$.

Lemma 6. Let $\left(\alpha_{i}\right)_{1 \leq i \leq n}$ be a sequence of complex numbers, and let $M=\left(m_{i j}\right)_{1 \leq i, j \leq n}$ be an $(n \times n)$-matrix with the entries

$$
m_{i j}=\frac{\alpha_{j}-\alpha_{i}}{j-i}, \quad 1 \leq i, j \leq n .
$$

Then

$$
\|M\|_{\mathcal{H}} \leq 2 \max _{1 \leq i \leq n-1}\left|\alpha_{i+1}-\alpha_{i}\right| \sum_{k=1}^{n-1} \frac{\log (n-k)+1}{k} .
$$

Proof. First, we check that the matrix $M$ can be represented as

$$
M=\sum_{k=1}^{n-1}\left[A^{(k)}+B^{(k)}\right],
$$

where $A^{(k)}=\left(a_{i j}^{(k)}\right)_{1 \leq i, j \leq n}$ and $B^{(k)}=\left(b_{i j}^{(k)}\right)_{1 \leq i, j \leq n}$ are the matrices with the entries

$$
\begin{aligned}
& a_{i j}^{(k)}= \begin{cases}\frac{\alpha_{i+k}-\alpha_{i+k-1}}{j-i} & \text { if } i+k \leq j \leq n, \\
0 & \text { otherwise, }\end{cases} \\
& b_{i j}^{(k)}= \begin{cases}\frac{\alpha_{j+k}-\alpha_{j+k-1}}{i-j} & \text { if } j+k \leq i \leq n, \\
0 & \text { otherwise. }\end{cases}
\end{aligned}
$$

It suffices to show that

$$
\frac{\alpha_{j}-\alpha_{i}}{j-i}=\sum_{k=1}^{n-1} a_{i j}^{(k)}
$$

for $i<j$. Since the elements $a_{i j}^{(k)}$ of $A^{(k)}$ may be different from zero for $k \leq j-i$ only, we have

$$
\begin{aligned}
\sum_{k=1}^{n-1} a_{i j}^{(k)} & =\sum_{k=1}^{j-i} \frac{\alpha_{i+k}-\alpha_{i+k-1}}{j-i} \\
& =\frac{1}{j-i}\left(\sum_{k=1}^{j-i} \alpha_{i+k}-\sum_{k=0}^{j-i-1} \alpha_{i+k}\right)=\frac{\alpha_{j}-\alpha_{i}}{j-i}=m_{i j} .
\end{aligned}
$$


Since the factors $\alpha_{i+k}-\alpha_{i+k-1}$ are constant in the rows of the matrix $A^{(k)}$, by Lemmas 3 and 5 we have

$$
\left\|A^{(k)}\right\|_{\mathcal{H}} \leq \max _{1 \leq i \leq n-1}\left|\alpha_{i+1}-\alpha_{i}\right| \cdot \frac{\log (n-k)+1}{k} .
$$

A similar estimate is true for the norm $\left\|B^{(k)}\right\|_{\mathcal{H}}$. The lemma follows from the representation (3).

Lemma 7. Suppose a sequence $\left(\alpha_{m}\right)_{m \geq 1}$ is such that $\alpha_{m}=\alpha_{n+1}$ for $m \geq n+1$, and the matrix $M=\left(m_{i j}\right)_{i, j \geq 1}$ is defined by

$$
m_{i j}= \begin{cases}\frac{\alpha_{n+1}-\alpha_{i}}{j-i} & \text { if } i=1, \ldots, n, j=n+1, n+2, \ldots, \\ 0 & \text { otherwise. }\end{cases}
$$

Then

$$
\|M\|_{\mathcal{H}} \leq \max _{1 \leq k \leq n-1}\left|\alpha_{k+1}-\alpha_{k}\right| \cdot(\log n+1)
$$

Proof. We write

$$
M=\sum_{k=1}^{n} M^{(k)}, \quad M^{(k)}=\left(m_{i j}^{(k)}\right)_{i, j \geq 1}
$$

where

$$
m_{i j}^{(k)}= \begin{cases}\frac{\alpha_{n-k+2}-\alpha_{n-k+1}}{j-i} & \text { if } i=1, \ldots, n-k+1, \\ 0 & j=n+1, n+2, \ldots, 2 \leq k+i \leq n+1, \\ 0 & \text { otherwise. }\end{cases}
$$

The representation (4) is obvious because

$$
\begin{aligned}
\sum_{k=1}^{n+1-i} m_{i j}^{(k)} & =\sum_{k=1}^{n+1-i}\left(\alpha_{n-k+2}-\alpha_{n-k+1}\right) \frac{1}{j-i} \\
& =\frac{1}{j-i}\left[\sum_{k=1}^{n+1-i} \alpha_{n-k+2}-\sum_{k=2}^{n+2-i} \alpha_{n-k+2}\right]=\frac{\alpha_{n+1}-\alpha_{i}}{j-i}
\end{aligned}
$$

Observe that only $n-k+1$ rows of the matrix $M^{(k)}$ are different from zero and that the factors $\alpha_{n-k+2}-\alpha_{n-k+1}$ are constant. Since, clearly, the multiplier norm of an orthogonal projection matrix is equal to one, by Lemma 4 we have

$$
\left\|M^{(k)}\right\|_{\mathcal{H}} \leq\left|\alpha_{n-k+2}-\alpha_{n-k+1}\right|\left\|F_{n, k}\right\|_{\mathcal{H}} \leq\left|\alpha_{n-k+2}-\alpha_{n-k+1}\right| \frac{1}{k} .
$$

Summing inequalities (5) and using the fact that $\sum_{k=1}^{n} \frac{1}{k}<\ln n+1$, we complete the proof.

\section{§2. Estimates FOR FUnCTIONS OF BOUNDED OPERATORS}

First we consider the case of commuting selfadjoint operators.

Theorem 8. Let $A$ and $A_{1}$ be commuting, bounded, selfadjoint operators, and let $f$ be a continuous function on an interval $[a, b]$ containing the spectra of $A$ and $A_{1}$. Let $\omega_{f}$ denote the modulus of continuity of $f$ on the interval $[a, b]$. Then

$$
\left\|f(A)-f\left(A_{1}\right)\right\| \leq \omega_{f}\left(\left\|A-A_{1}\right\|\right) .
$$


The immediate proof of this theorem employs an idea of V. V. Peller proposed for the case of $\operatorname{Lip}(\alpha)$-functions. Since two commuting selfadjoint operators have a spectral measure on the joint spectrum $\sigma\left(A, A_{1}\right)$, we have

$$
\begin{aligned}
\left\|f(A)-f\left(A_{1}\right)\right\| & =\sup _{x, y \in \sigma\left(A, A_{1}\right)}|f(x)-f(y)| \\
& =\sup \left\{|f(x)-f(y)|:|x-y| \leq \sup _{z, t \in \sigma\left(A, A_{1}\right)}|z-t|\right\} \\
& =\sup _{|x-y| \leq\left\|A-A_{1}\right\|}|f(x)-f(y)|=\omega_{f}\left(\left\|A-A_{1}\right\|\right) .
\end{aligned}
$$

Remark. The conclusion of Theorem 8 remains true in the case of unbounded commuting selfadjoint operators $A$ and $A_{1}$ such that the operator $A-A_{1}$ is bounded.

Now, consider the following elementary case. Let $A$ and $B$ be two selfadjoint operators on $H$ with finite spectra. Assume that the eigenvalues of $A$ and $B$ are among real numbers $\nu_{1}, \ldots, \nu_{n}$ such that $\nu_{i+1}-\nu_{i}=d>0, i=1, \ldots, n-1$. Next, let $f$ be a continuous function on an interval containing all points $\nu_{i}$. Let $P_{i}$ and $Q_{i}$ be the orthogonal spectral projections of the operators $A$ and $B$ (respectively) associated with the eigenvalue $\nu_{i}$ (if $\nu_{i}$ is not an eigenvalue, we assume that the projection is zero). Since $P_{i} A=A P_{i}=\nu_{i} P_{i}$ and $Q_{j} B=B Q_{j}=\nu_{j} Q_{j}$, we have

$$
P_{i} Q_{j}=\frac{P_{i}(A-B) Q_{j}}{\nu_{i}-\nu_{j}} .
$$

Therefore,

$$
\begin{aligned}
f(A)-f(B) & =\sum_{i=1}^{n} \sum_{j=1}^{n} \frac{f\left(\nu_{j}\right)-f\left(\nu_{i}\right)}{\nu_{j}-\nu_{i}} P_{i}(A-B) Q_{j} \\
& =\frac{1}{d} \sum_{i=1}^{n} \sum_{i=1}^{n} \frac{f\left(\nu_{j}\right)-f\left(\nu_{i}\right)}{j-i} P_{i}(A-B) Q_{j} .
\end{aligned}
$$

(By definition, a fraction is equal to zero if the numerator is zero.)

Note that this representation of the operator $f(A)-f(B)$ is a particular case of a double operator integral (see [3]). Of course, $f(A)-f(B)$ is a matrix with operator entries, but the orthogonality property of projections implies that the norms of such matrices and the norms of numerical matrices are calculated similarly.

Theorem 9. Suppose that the operators $A$ and $B$ satisfy the above conditions. Then

$$
\|f(A)-f(B)\| \leq \frac{2}{d} \max _{1 \leq i \leq n}\left|f\left(\nu_{i+1}\right)-f\left(\nu_{i}\right)\right| \sum_{k=1}^{n-1} \frac{\log (n-k)+1}{k}\|A-B\| .
$$

Proof. Obviously, the operator $f(A)-f(B)$ may be regarded as the operator matrix $\frac{1}{d} \cdot M \circ T$, where

$$
M=\left(\frac{f\left(\nu_{j}\right)-f\left(\nu_{i}\right)}{j-i}\right)_{1 \leq i, j \leq n}, \quad T=\left(P_{i}(A-B) Q_{j}\right)_{1 \leq i, j \leq n} .
$$

Since $\|M \circ T\| \leq\|M\|_{\mathcal{H}}\|T\|$, the theorem follows from Lemma 6 .

Now we are able to prove the following theorem in the general case of two bounded selfadjoint operators.

Theorem 10. Let $A$ and $B$ be bounded selfadjoint operators on a separable Hilbert space. Suppose that $f$ is a continuous function on an interval $[a, b]$ containing the spectra of $A$ 
and B. Let $\omega_{f}$ denote the modulus of continuity of $f$. Then

$$
\|f(A)-f(B)\| \leq 4\left[\log \left(\frac{b-a}{\|A-B\|}+1\right)+1\right]^{2} \cdot \omega_{f}(\|A-B\|) .
$$

Proof. We put $d=\|A-B\|$ and split the interval $[a, b]$ by the points $\left\{\nu_{i}\right\}_{1 \leq i \leq n}, \nu_{i}=$ $a+\left(i-\frac{1}{2}\right) d$. Clearly, $\nu_{n-1}<b, \nu_{n} \geq b, \nu_{i+1}-\nu_{i}=d$, and $2 \leq n \leq \frac{b-a}{\|A-B\|}+1$.

We introduce a piecewise constant function $\varphi$ defined on the interval $[a, b]$ as follows: $\varphi(t)=\nu_{i}$ if $\nu_{i}-\frac{d}{2} \leq t<\nu_{i}+\frac{d}{2}$. Next, denote $A_{1}=\varphi(A), B_{1}=\varphi(B)$. Clearly, $A_{1}$ commutes with $A, B_{1}$ commutes with $B$, and $\left\|A-A_{1}\right\| \leq \frac{d}{2},\left\|B-B_{1}\right\| \leq \frac{d}{2},\left\|A_{1}-B_{1}\right\| \leq$ $2\|A-B\|=2 d$.

By Theorem 8, we have

$$
\left\|f(A)-f\left(A_{1}\right)\right\| \leq \omega_{f}\left(\left\|A-A_{1}\right\|\right) \leq \omega_{f}\left(\frac{d}{2}\right)=\omega_{f}\left(\frac{\|A-B\|}{2}\right) .
$$

Similarly,

$$
\left\|f(B)-f\left(B_{1}\right)\right\| \leq \omega_{f}\left(\frac{\|A-B\|}{2}\right) .
$$

Therefore,

$$
\|f(A)-f(B)\| \leq 2 \omega_{f}\left(\frac{\|A-B\|}{2}\right)+\left\|f\left(A_{1}\right)-f\left(B_{1}\right)\right\| .
$$

Since the spectra of the operators $A_{1}$ and $B_{1}$ coincide with the set $\nu_{1}, \ldots, \nu_{n}$, we can apply Theorem 9:

$$
\left\|f\left(A_{1}\right)-f\left(B_{1}\right)\right\| \leq \frac{2}{d} \max _{1 \leq i \leq n-1}\left|f\left(\nu_{i+1}\right)-f\left(\nu_{i}\right)\right| \sum_{k=1}^{n-1} \frac{\ln (n-k)+1}{k} \cdot\left\|A_{1}-B_{1}\right\| .
$$

Since $\left\|A_{1}-B_{1}\right\| \leq 2 d$ and $\max _{1 \leq i \leq n-1}\left|f\left(\nu_{i+1}\right)-f\left(\nu_{i}\right)\right| \leq \omega_{f}(d)=\omega_{f}(\|A-B\|)$, we have

$$
\left\|f\left(A_{1}\right)-f\left(B_{1}\right)\right\| \leq 4 \cdot \omega_{f}(\|A-B\|) \cdot \sum_{k=1}^{n-1} \frac{\log (n-k)+1}{k} .
$$

An elementary calculation shows that $\sum_{k=1}^{n-1} \frac{\log (n-k)+1}{k} \leq \log ^{2} n+\log n$. Since the function $\omega_{f}$ is monotone increasing and $n<\frac{b-a}{\|A-B\|}+1$, the theorem follows from inequalities (7), (8).

Remark 1. Clearly, if $f \in \operatorname{Lip}(\alpha), 0<\alpha \leq 1$, then inequality (6) leads to

$$
\|f(A)-f(B)\| \leq 4[f] \cdot\left[\log \left(\frac{b-a}{\|A-B\|}+1\right)+1\right]^{2}\|A-B\|^{\alpha},
$$

where $[f]$ is the Lipschitz constant of $f$ on the interval $[a, b]$.

Remark 2. It is easily seen that if $A$ and $K_{n}$ are bounded selfadjoint operators such that $\left\|K_{n}\right\| \rightarrow 0$, and $f$ is a continuous function, then $\lim _{n \rightarrow \infty}\left\|f\left(A+K_{n}\right)-f(A)\right\|=0$. This follows from the Weierstrass theorem and inequality (1) for polynomials. So, the estimate in Theorem 10 is not sharp. But it is effective for a large class of functions whose modulus of continuity decreases faster than $\frac{1}{\log ^{2} \delta}$.

On the other hand, the presence of a growing factor in (6) is inevitable. In 13, it was shown that there exists a function $f \in \operatorname{Lip}(1)$ and selfadjoint operators $\left\{A_{n}\right\},\left\{B_{n}\right\}$ such that their spectra are contained in $[0,1],\left\|B_{n}-A_{n}\right\| \rightarrow 0$, but $\frac{\left\|f\left(B_{n}\right)-f\left(A_{n}\right)\right\|}{\left\|B_{n}-A_{n}\right\|} \rightarrow \infty$. 
Remark 3. It is easy to show that if $T$ is a bounded operator and two operators $A$ and $B$ satisfy the above conditions, then

$$
f(A) T-T f(B)=\frac{1}{d} \sum_{i=1}^{n} \sum_{j=1}^{n} \frac{f\left(\nu_{j}\right)-f\left(\nu_{i}\right)}{j-i} P_{i}(A T-T B) Q_{j} .
$$

Therefore, minor modifications of the proof of Theorem 10 lead to the following theorem on commutators.

Theorem 11. Let $A, B$ and $T$ be bounded operators on a separable Hilbert space. Suppose that $A$ and $B$ are selfadjoint and that $f$ is a continuous function on an interval $[a, b]$ containing the spectra of $A$ and $B$. Let $\omega_{f}$ denote the modulus of continuity of $f$. Then

$$
\|f(A) T-T f(B)\| \leq 4\|T\| \cdot\left[\log \left(\frac{(b-a)\|T\|}{\|A T-T B\|}+1\right)+1\right]^{2} \cdot \omega_{f}\left(\frac{\|A T-T B\|}{\|T\|}\right) .
$$

\section{§3. Estimates FOR FUnCTIONS OF UNBOUNDED OPERATORS}

Let $A_{1}$ and $B_{1}$ be unbounded selfadjoint operators on a separable Hilbert space. Assume that the operator $A_{1}-B_{1}$ is bounded (in particular, this implies that the operators $A_{1}$ and $B_{1}$ are defined on the same domain). Suppose that the spectra of the operators $A_{1}$ and $B_{1}$ coincide and consist of eigenvalues. More precisely, $\sigma\left(A_{1}\right)=\sigma\left(B_{1}\right) \subset\left\{\lambda_{i}\right\}_{i \in \mathbb{Z}}$, $\lambda_{i}-\lambda_{i-1}=d>0$ for all $i \in \mathbb{Z}$.

Next, let $[a, b]$ be an interval and $f$ a continuous function on $\mathbb{R}$ such that $f(x)=f(a)$, $x \leq a$ and $f(x)=f(b), x \geq b$. Let $\omega_{f}$ denote the modulus of continuity of the function $f$ on $\mathbb{R}$. Clearly, $\omega_{f}$ coincides with the modulus of continuity of $f$ on the interval $[a, b]$. Assume that $\lambda_{1}<a, \lambda_{2} \geq a$ and that $n$ is an integer such that $\lambda_{n-1}<b, \lambda_{n} \geq b$. Clearly, $2 \leq n \leq \frac{b-a}{d}+1$.

Lemma 12. Under the above conditions, we have

$$
\left\|f\left(A_{1}\right)-f\left(B_{1}\right)\right\| \leq \frac{2}{d} \omega_{f}(d)\left\|A_{1}-B_{1}\right\|\left(\log ^{2} n+3 \log n+3\right) .
$$

Proof. We identify the operator $A_{1}-B_{1}$ with the operator matrix $A_{1}-B_{1}=\left(T_{i j}\right)_{i, j \geq 1}$ whose entries $T_{i j}=P_{i}\left(A_{1}-B_{1}\right) Q_{j}, P_{i}, Q_{j}$ are the orthogonal projections of the operators $A_{1}$ and $B_{1}$ associated with the eigenvalues $\lambda_{i}, \lambda_{j}$. Clearly,

$$
f\left(B_{1}\right)-f\left(A_{1}\right)=\frac{1}{d} \cdot \sum_{i=-\infty}^{\infty} \sum_{j=-\infty}^{\infty} \frac{f\left(\lambda_{j}\right)-f\left(\lambda_{i}\right)}{j-i} T_{i j} .
$$

Now we represent the sum in (10) as follows:

$$
\sum_{i=-\infty}^{\infty} \sum_{j=-\infty}^{\infty} \frac{f\left(\lambda_{j}\right)-f\left(\lambda_{i}\right)}{j-i} T_{i j}=S_{1}+\cdots+S_{9}
$$

where

$$
\begin{aligned}
& S_{1}=\sum_{i=1}^{n} \sum_{j=1}^{n} \frac{f\left(\lambda_{j}\right)-f\left(\lambda_{i}\right)}{j-i} T_{i j}, \\
& S_{2}=\sum_{i=1}^{n} \sum_{j=n+1}^{\infty} \frac{f\left(\lambda_{j}\right)-f\left(\lambda_{i}\right)}{j-i} T_{i j}=\sum_{i=1}^{n} \sum_{j=n+1}^{\infty} \frac{f\left(\lambda_{n}\right)-f\left(\lambda_{i}\right)}{j-i} T_{i j}, \\
& S_{3}=\sum_{i=1}^{n} \sum_{j=-\infty}^{0} \frac{f\left(\lambda_{j}\right)-f\left(\lambda_{i}\right)}{j-i} T_{i j}=\sum_{i=1}^{n} \sum_{j=-\infty}^{0} \frac{f\left(\lambda_{i}\right)-f\left(\lambda_{1}\right)}{i-j} T_{i j},
\end{aligned}
$$




$$
\begin{aligned}
& S_{4}=\sum_{i=-\infty}^{0} \sum_{j=1}^{n} \frac{f\left(\lambda_{j}\right)-f\left(\lambda_{1}\right)}{j-i} T_{i j}, \\
& S_{5}=\sum_{i=-\infty}^{0} \sum_{j=-\infty}^{0} \frac{f\left(\lambda_{j}\right)-f\left(\lambda_{i}\right)}{j-i} T_{i j}=\mathbf{0}, \\
& S_{6}=\sum_{i=-\infty}^{\infty} \sum_{j=n+1}^{\infty} \frac{f\left(\lambda_{n}\right)-f\left(\lambda_{1}\right)}{j-i} T_{i j}, \\
& S_{7}=\sum_{i=n+1}^{\infty} \sum_{j=1}^{n} \frac{f\left(\lambda_{j}\right)-f\left(\lambda_{n}\right)}{j-i} T_{i j}, \\
& S_{8}=\sum_{i=n+1}^{\infty} \sum_{j=-\infty}^{0} \frac{f\left(\lambda_{1}\right)-f\left(\lambda_{n}\right)}{j-i} T_{i j} T_{i j}, \\
& S_{9}=\sum_{i=n+1}^{\infty} \sum_{j=n+1}^{\infty} \frac{f\left(\lambda_{j}\right)-f\left(\lambda_{i}\right)}{j-i} T_{i j}=\mathbf{0 .} .
\end{aligned}
$$

We estimate each term in (11) separately.

By Lemma 6,

$$
\begin{aligned}
\left\|S_{1}\right\| & \leq 2\left\|A_{1}-B_{1}\right\| \max _{1 \leq i \leq n}\left|f\left(\lambda_{i+1}\right)-f\left(\lambda_{i}\right)\right| \sum_{k=1}^{n-1} \frac{\log (n-k)+1}{k} \\
& \leq 2\left\|A_{1}-B_{1}\right\| \omega_{f}(d)\left(\log ^{2} n+\log n\right) .
\end{aligned}
$$

Observe that estimates for the operators $S_{2}, S_{3}, S_{4}$, and $S_{7}$ are similar, and the same is true for $S_{6}$ and $S_{8}, S_{5}=S_{9}=\mathbf{0}$. Thus, it suffices to estimate the operators $S_{2}$ and $S_{6}$.

By Lemma 7,

$$
\left\|S_{2}\right\| \leq\left\|A_{1}-B_{1}\right\| \max _{1 \leq k \leq n}\left|f\left(\lambda_{k+1}\right)-f\left(\lambda_{k}\right)\right|(\log n+1) \leq\left\|A_{1}-B_{1}\right\| \omega_{f}(d)(\log n+1) .
$$

For the operator $S_{6}$ we have

$$
S_{6}=\left(f\left(\lambda_{n}\right)-f\left(\lambda_{1}\right)\right) \sum_{i=0}^{\infty} \sum_{j=n+1}^{\infty} \frac{1}{j+i} T_{-i, j} .
$$

So, we need to estimate the multiplier norm of the matrix

$$
M_{n}=\left(\begin{array}{cccccc}
0 & \ldots & 0 & \frac{1}{n+1} & \frac{1}{n+2} & \ldots \\
0 & \ldots & 0 & \frac{1}{n+2} & \frac{1}{n+3} & \ldots \\
0 & \ldots & 0 & \frac{1}{n+3} & \frac{1}{n+4} & \ldots \\
\ldots & \ldots & \ldots & \ldots & \ldots & \ldots \\
\ldots & \ldots & \ldots & \ldots & \ldots & \ldots \\
\ldots & \ldots & \ldots & \ldots & \ldots & \ldots
\end{array}\right) .
$$

Clearly, $\left\|M_{n}\right\|_{\mathcal{H}} \leq \frac{1}{n+1}$. Therefore,

$$
\begin{aligned}
\left\|S_{6}\right\| & \leq\left\|A_{1}-B_{1}\right\| \frac{\left|f\left(\lambda_{n}\right)-f\left(\lambda_{1}\right)\right|}{n+1}=\left\|A_{1}-B_{1}\right\| \frac{1}{n+1}\left|\sum_{k=1}^{n-1}\left(f\left(\lambda_{k+1}\right)-f\left(\lambda_{k}\right)\right)\right| \\
& \leq\left\|A_{1}-B_{1}\right\| \max _{1 \leq k \leq n-1}\left|f\left(\lambda_{k+1}\right)-f\left(\lambda_{k}\right)\right| \leq\left\|A_{1}-B_{1}\right\| \omega_{f}(d) .
\end{aligned}
$$


Finally, formulas (10) and (11) lead to

$$
\left\|f\left(A_{1}\right)-f\left(B_{1}\right)\right\| \leq \frac{2}{d} \omega_{f}(d)\left\|A_{1}-B_{1}\right\|\left(\log ^{2} n+3 \log n+3\right) .
$$

The lemma is proved.

In what follows we always assume that the function $f$ is defined on the real line and satisfies the following condition:

(*) there exist $\beta>2$ and $M>0$ such that $|f(t)| \leq M|t|$ for $|t|>\beta$.

We cover the real line $\mathbb{R}$ by the set of intervals $\left.\left.\Delta_{i}=\right] \beta_{i}, \beta_{i+1}\right], i \in \mathbb{Z}$, such that $\beta_{0}=-\beta$, $\beta_{1}=\beta>2$, and $\beta_{i+1}-\beta_{i} \geq 1, i \neq 0$. Accordingly, consider the set of functions $\left\{f_{i}\right\}_{i \in \mathbb{Z}}$ defined on the real line by

$$
f_{i}(t)= \begin{cases}f(t) & \text { if } \beta_{i} \leq t \leq \beta_{i+1}, \\ f\left(\beta_{i}\right) & \text { if } t \leq \beta_{i} \\ f\left(\beta_{i+1}\right) & \text { if } t \geq \beta_{i+1} .\end{cases}
$$

Lemma 13. Let $A$ and $B$ be two selfadjoint operators with bounded difference. Suppose that a vector $x$ belongs to the domain of the operator $A$ (this means automatically that $x$ also belongs to the domain of the operator $B)$, and let $f, f_{i}$ be the functions defined above. Then

$$
((f(A)-f(B)) x, x)=\sum_{i=-\infty}^{\infty}\left(\left(f_{i}(A)-f_{i}(B)\right) x, x\right) .
$$

Proof. First, observe that $x$ belongs to the domain of all operators $f_{i}(A)$ and $f_{i}(B)$. We denote by $E_{A}$ and $E_{B}$ the spectral measures of the operators $A$ and $B$ (respectively) and introduce the function $\varphi$ defined on the real line by $\varphi(\lambda)=\left(\left(E_{A}(\lambda)-E_{B}(\lambda)\right) x, x\right)$. Clearly, $\lim _{\lambda \rightarrow-\infty(\infty)} \varphi(\lambda)=0$. We have

$$
((f(A)-f(B)) x, x)=\int_{-\infty}^{\infty} f(\lambda) d \varphi=\sum_{i=-\infty}^{\infty} \int_{\beta_{i}}^{\beta_{i+1}} f_{i}(\lambda) d \varphi=\sum_{i=-\infty}^{\infty} S_{i}
$$

where

$$
\begin{aligned}
S_{i} & =\int_{\beta_{i}}^{\beta_{i+1}} f_{i}(\lambda) d \varphi=\int_{-\infty}^{\infty} f_{i}(\lambda) d \varphi-\left[f_{i}\left(\beta_{i+1}\right) \int_{\beta_{i+1}}^{\infty} d \varphi+f_{i}\left(\beta_{i}\right) \int_{-\infty}^{\beta_{i}} d \varphi\right] \\
& =\int_{-\infty}^{\infty} f_{i}(\lambda) d \varphi+f\left(\beta_{i+1}\right) \varphi\left(\beta_{i+1}\right)-f\left(\beta_{i}\right) \varphi\left(\beta_{i}\right) .
\end{aligned}
$$

We show that $\lim _{i \rightarrow-\infty(\infty)} f\left(\beta_{i}\right) \varphi\left(\beta_{i}\right)=0$.

Let $i \rightarrow+\infty$. We may assume that $\beta_{i}>0$. Since $\left(E_{A}\left(\beta_{i}\right) x, x\right)=\int_{-\infty}^{\beta_{i}} d\left(E_{A}(\lambda) x, x\right)$ and $\int_{-\infty}^{\infty} d\left(E_{A}(\lambda) x, x\right)=\int_{-\infty}^{\infty} d\left(E_{B}(\lambda) x, x\right)=\|x\|^{2}$, we have

$$
\begin{aligned}
f\left(\beta_{i}\right) \varphi\left(\beta_{i}\right) & \left.=f\left(\beta_{i}\right)\left[\left(E_{A}\left(\beta_{i}\right) x, x\right)-E_{B}\left(\beta_{i}\right) x, x\right)\right] \\
& =-f\left(\beta_{i}\right) \int_{\beta_{i}}^{\infty} d\left(E_{A}(\lambda) x, x\right)+f\left(\beta_{i}\right) \int_{\beta_{i}}^{\infty} d\left(E_{B}(\lambda) x, x\right) .
\end{aligned}
$$

Using the assumptions imposed on the function $f$, we obtain

$$
\left|f\left(\beta_{i}\right)\right| \int_{\beta_{i}}^{\infty} d\left(E_{A}(\lambda) x, x\right) \leq M \beta_{i} \int_{\beta_{i}}^{\infty} d\left(E_{A}(\lambda) x, x\right) \leq M \int_{\beta_{i}}^{\infty} \lambda d\left(E_{A}(\lambda) x, x\right) \longrightarrow 0 .
$$

A similar argument can be applied to the case where $i \rightarrow-\infty$. Now, clearly,

$$
\sum_{i=-\infty}^{\infty}\left[f\left(\beta_{i+1}\right) \varphi\left(\beta_{i+1}\right)-f\left(\beta_{i}\right) \varphi\left(\beta_{i}\right)\right]=0
$$


So,

$$
\begin{aligned}
((f(A)-f(B)) x, x) & =\sum_{i=-\infty}^{\infty} S_{i} \\
& =\sum_{i=-\infty}^{\infty}\left[\int_{-\infty}^{\infty} f_{i}(\lambda) d \varphi+f\left(\beta_{i+1}\right) \varphi\left(\beta_{i+1}\right)-f\left(\beta_{i}\right) \varphi\left(\beta_{i}\right)\right] \\
& =\sum_{i=-\infty}^{\infty}\left(\left(f_{i}(A)-f_{i}(B)\right) x, x\right) .
\end{aligned}
$$

The lemma follows.

Corollary. Obviously, if the operator $f(A)-f(B)$ is bounded, then for the functions defined above we have

$$
\|f(A)-f(B)\| \leq \sum_{i=-\infty}^{\infty}\left\|f_{i}(A)-f_{i}(B)\right\| .
$$

Now we need an additional assumption on the modulus of continuity of the functions under consideration. Assume that the function $f$ on the real line satisfies condition $(*)$. Denote by $\omega_{f, 0}$ the modulus of continuity of $f$ on the interval $[-\beta, \beta]$ and by $\omega_{f, t}$ the modulus of continuity of $f$ on the set $\{x \in \mathbb{R}:|x| \geq t>\beta\}$. We assume that, for $0<d \leq 1$

$$
\omega_{f, t}(d) \leq C_{1} \frac{\omega_{f, 0}(d)}{(\log |t|)^{3}}, \quad C_{1}>0 .
$$

Note that for $f \in C^{(1)}(\mathbb{R})$, condition $(* *)$ is fulfilled if $\left|f^{\prime}(x)\right| \leq \frac{\text { const }}{(\log |x|)^{3}},|x| \geq t>\beta$.

Lemma 14. Let $A_{1}$ and $B_{1}$ be operators as in Lemma 12 and suppose that the function $f$ satisfies conditions $(*)$ and $(* *)$. Then the operator $f\left(A_{1}\right)-f\left(B_{1}\right)$ is bounded, and there is a positive constant $D_{1}$ such that

$$
\left\|f\left(A_{1}\right)-f\left(B_{1}\right)\right\| \leq D_{1} \frac{1}{d} \omega_{f, 0}(d)\left\|A_{1}-B_{1}\right\| \log ^{2}\left(1+\frac{1}{d}\right) .
$$

Proof. We apply Lemma 12 to the intervals $[-\beta, \beta]$ and $\left[\beta_{i}, \beta_{i+1}\right]$. We have

$$
\left\|f_{0}\left(A_{1}\right)-f_{0}\left(B_{1}\right)\right\| \leq \frac{2}{d} \omega_{f, 0}(d)\left\|A_{1}-B_{1}\right\|\left(\log ^{2} n_{0}+3 \log n_{0}+3\right)
$$

and

$$
\left\|f_{i}\left(A_{1}\right)-f_{i}\left(B_{1}\right)\right\| \leq \frac{2}{d} \omega_{f, i}(d)\left\|A_{1}-B_{1}\right\|\left(\log ^{2} n_{i}+3 \log n_{i}+3\right) .
$$

Here $\omega_{f, i}, i \neq 0$, is the modulus of continuity of $f$ on the interval $\left[\beta_{i}, \beta_{i+1}\right], n_{i} \leq$ $1+\frac{\beta_{i+1}-\beta_{i}}{d}$ for $i \neq 0$, and $2 \leq n_{0} \leq 1+\frac{2 \beta}{d}$.

Suppose for convenience that the intervals $\Delta_{i}$ and $\Delta_{-i}$ are symmetric to each other, and put $\beta_{i}=2^{i^{2}}+\beta, i \geq 1$. Then $\beta_{i+1}-\beta_{i}<2^{(i+1)^{2}}$. Moreover, since $2 \leq n_{0} \leq 1+\frac{2 \beta}{d}$ and $n_{i} \leq 1+\frac{\beta_{i+1}-\beta_{i}}{d}$, and since $\log \left(1+\frac{b}{a}\right) \leq \log b+\log \left(1+\frac{1}{a}\right)$ for $a>0, b>1$, we have

$$
\begin{gathered}
\log ^{2} n_{0}+3 \log n_{0}+3 \leq 7 \log ^{2} n_{0}, \quad \log n_{0} \leq \log (2 \beta)+\log \left(1+\frac{1}{d}\right), \\
\log n_{i} \leq \log \left(\beta_{i+1}-\beta_{i}\right)+\log \left(1+\frac{1}{d}\right) \leq(i+1)^{2}+\log \left(1+\frac{1}{d}\right) .
\end{gathered}
$$

Next, the function $f$ satisfies condition $(* *)$. So,

$$
\omega_{f, i}(d) \leq C_{1} \frac{\omega_{f, 0}(d)}{\log ^{3} \beta_{i}} \leq C_{1} \frac{\omega_{f, 0}(d)}{\log ^{3}\left(2^{i^{2}}\right)}=C_{1} \frac{\omega_{f, 0}}{i^{6}}
$$


Summing inequalities (12) and (13) over $i \geq 0$, we get

$$
\begin{aligned}
\sum_{i=1}^{\infty} \| f_{i} & \left(A_{1}\right)-f_{i}\left(B_{1}\right)\|+\| f_{0}\left(A_{1}\right)-f_{0}\left(B_{1}\right) \| \\
\leq & \frac{2}{d}\left\|A_{1}-B_{1}\right\| \\
& \times\left[\sum_{i=1}^{\infty} \omega_{f, i}(d)\left(\log ^{2} n_{i}+3 \log n_{i}+3\right)+7 \omega_{f, 0}(d)\left(\log (2 \beta)+\log \left(1+\frac{1}{d}\right)\right)^{2}\right] \\
\leq & \frac{2}{d}\left\|A_{1}-B_{1}\right\| \omega_{f, 0}(d) \cdot\left[7\left(\log (2 \beta)+\log \left(1+\frac{1}{d}\right)\right)^{2}\right] \\
& +\sum_{i=1}^{\infty} C_{1} \frac{\left.\left[(i+1)^{2}+\log \left(1+\frac{1}{d}\right)\right]^{2}+3(i+1)^{2}+3 \log \left(1+\frac{1}{d}\right)+3\right]}{i^{6}}<\infty .
\end{aligned}
$$

On the intervals $\Delta_{-i}, i \geq 1$, the result is similar.

Now it is obvious that there is a positive constant $D_{1}$ depending on $\beta$ and $C_{1}$ such that

$$
\left\|f_{0}\left(A_{1}\right)-f_{0}\left(B_{1}\right)\right\|+\sum_{i \neq 0}\left\|f_{i}\left(A_{1}\right)-f_{i}\left(B_{1}\right)\right\| \leq D_{1} \frac{1}{d} \omega_{f, 0}(d)\left\|A_{1}-B_{1}\right\| \log ^{2}\left(1+\frac{1}{d}\right) .
$$

The lemma follows from the corollary to Lemma 13.

Theorem 15. Let $A$ and $B$ be unbounded selfadjoint operators on a separable Hilbert space. Assume that the operator $A-B$ is bounded and that the function $f$ on the real line satisfies conditions $(*)$ and $(* *)$. Then the operator $f(A)-f(B)$ is bounded, and there is a positive constant $D$ such that

$$
\|f(A)-f(B)\| \leq D \omega_{f, 0}(\|A-B\|) \log ^{2}\left(1+\frac{1}{\|A-B\|}\right) .
$$

Proof. The proof of this theorem is similar to that of Theorem 10. We consider a set $\left\{\lambda_{i} \in \mathbb{R}: \lambda_{i+1}-\lambda_{i}=d>0\right\}_{i \in \mathbb{Z}}$ of real numbers and define a piecewise constant function $\varphi$ on $\mathbb{R}$ as follows: $\varphi(t)=\lambda_{i}$ if $\lambda_{i}-\frac{d}{2} \leq t<\lambda_{i}+\frac{d}{2}, i \in \mathbb{Z}$. Put $A_{1}=\varphi(A), B_{1}=\varphi(B)$. Then, by Lemma 13, the operator $f\left(A_{1}\right)-f\left(B_{1}\right)$ is bounded. Therefore, setting $\|A-B\|=d \leq 1$ and arguing as in the proof of Theorem 10, we obtain $\left\|A_{1}-B_{1}\right\| \leq 2\|A-B\|$ and

$$
\|f(A)-f(B)\| \leq 2 \omega_{f}\left(\frac{\|A-B\|}{2}\right)+\left\|f\left(A_{1}\right)-f\left(B_{1}\right)\right\| .
$$

Note that $\omega_{f}\left(\frac{\|A-B\|}{2}\right) \leq D_{2} \omega_{f, 0}\left(\frac{\|A-B\|}{2}\right) \leq D_{2} \omega_{f, 0}(\|A-B\|)$, where $D_{2}=\max \left\{1, \frac{C_{1}}{\log ^{3} \beta}\right\}$ $\leq \max \left\{1, C_{1}\right\}$. The theorem follows from Lemma 14 if we put $D=4 \max \left\{D_{1}, D_{2}\right\}$.

Corollary 16. Let $f$ be a $\operatorname{Lip}(\alpha)$-function on $\mathbb{R}, 0<\alpha \leq 1$. We denote by $[f]_{t}$ the Lipschitz constant of $f$ on the set $\{x \in \mathbb{R}:|x|>t>\beta\}$ and assume that $[f]_{t} \leq \frac{c}{(\log |t|)^{3}}$, $c>0$. Then $f$ is an operator continuous function, and there is a positive constant $L$ such that

$$
\|f(A)-f(B)\| \leq L \log ^{2}\left(1+\frac{1}{\|A-B\|}\right)\|A-B\|^{\alpha}, \quad L>0 .
$$

Clearly, the constant $L$ depends on $c$ and on $[f]_{\beta}$, where $[f]_{\beta}$ is the Lipschitz constant of $f$ on the interval $[-\beta, \beta]$.

Now we formulate a condition sufficient for operator continuity. Suppose that

$$
\omega_{f, 0}(d) \leq \frac{C_{2}}{\log ^{2+\gamma}\left(1+\frac{1}{d}\right)}, \quad C_{2}>0, \quad \gamma>0 .
$$


Corollary 17. Any continuous function $f$ on $\mathbb{R}$ satisfying conditions $(*)-(* * *)$ is operator continuous.

\section{ACKNOWLEDGEMENTS}

This research was done with the financial support of the European Research and Training Network "Classical Analysis", the Bordeaux node. Yu. Farforovskaya is grateful to the University of Bordeaux-1 for hospitality during her visits in 2005 and 2006, which enabled her to complete this research.

The authors thank V. V. Peller, whose help was as an unfailing source of mathematical information, and V. Shulman for very helpful discussions.

\section{REFERENCES}

[1] V. V. Peller, Multiple operator integrals and higher operator derivatives, J. Funct. Anal. 233 (2006), no. 2, 515-544. MR2214586(2008e:47056)

[2] _ Hankel operators in the perturbation theory of unbounded selfadjoint operators, Analysis and Partial Differential Equations, Lecture Notes in Pure and Appl. Math., vol. 122, Dekker, New York, 1990, pp. 529-544. MR1044807 (92d:47037)

[3] M. Sh. Birman and M. Z. Solomyak, Double Stieltjes operator integrals, Probl. Mat. Fiz., No. 1, Spectral Theory and Wave Processes, Leningrad. Univ., Leningrad, 1966, pp. 33-67; English transl., Topics in Math. Phys., vol. 1, Consultants Bureau [Plenum Publ. Corporation], New York, 1967, pp. 25-54. MR0209872(35:767b)

[4] G. Bennett, Schur multipliers, Duke Math. J. 44 (1977), 603-639. MR0493490 (58:12490)

[5] L. N. Nikol'skaya and Yu. B. Farforovskaya, Toeplitz and Hankel matrices as Hadamard-Schur multipliers, Algebra i Analiz 15 (2003), no. 6, 141-160; English transl., St. Petersburg Math. J. 15 (2004), no. 6, 915-928. MR2044634 (2005a:47050)

[6] V. I. Matsaev, A class of completely continuous operators, Dokl. Akad. Nauk SSSR 139 (1961), no. 3, 548-551; English transl., Soviet Math. Dokl. 2 (1961), 972-975. MR0131769 (24:A1617)

[7] G. Polya, Remarks on characteristic functions, Proceedings of the Berkeley Symposium on Mathematical Statistics and Probability, 1945, 1946, Univ. California Press, Berkeley-Los Angeles, 1949, pp. 115-123. MR0028541 (10:463c)

[8] S. Bochner, Monotone Funktionen, Stieltjessche Integrale und harmonische Analyse, Math. Ann. 108 (1933), 378-410. MR.1512856

[9] G. Herglotz, Über Potenzreihen mit positivem, reellem Teil im Einheitskreis, S.-B. Sächs. Akad. Wiss. 63 (1911), 501-511.

[10] J. Schur, Bemerkungen zur Theorie der beschränkten Bilinearformen mit unendlich vielen Veränderlichen, J. Reine Angew. Math. 140 (1911), 1-28.

[11] H. Helson, Harmonic analysis, Addison-Wesley Publ. Co., Reading, MA, 1983. MR0729682 (85e:43001)

[12] Yu. B. Farforovskaya, Double operator integrals and their estimates in the uniform norm, Zap. Nauchn. Sem. S.-Peterburg. Otdel. Mat. Inst. Steklov. (POMI) 232 (1996), 148-173; English transl., J. Math. Sci. (New York) 92 (1998), no. 1, 3640-3656. MR.1464432 (98i:47018)

[13] _ An estimate of the norm $|f(B)-f(A)|$ for selfadjoint operators $A$ and $B$, Zap. Nauchn. Sem. Leningrad. Otdel. Mat. Inst. Steklov. (LOMI) 56 (1976), 143-162; English transl., J. Soviet Math. 14 (1980), no. 2, 1133-1149. MR0477850(57:17352)

[14] _ Estimates for commutators of normal operators, Algebra i Analiz 11 (1999), no. 4, 204-221; English transl., St. Petersburg Math. J. 11 (2000), no. 4, 687-699. MR1713939 (2000j:47040)

[15] Yu. B. Farforovskaya and L. Nikolskaia, An inequality for commutators of normal operators, Acta Sci. Math. (Szeged) 71 (2005), 751-765. MR2206607 (2006k:47043)

Mathematics Department, State University of Telecommunication, St. Petersburg, Russia E-mail address: rabk@sut.ru

Institut de Mathématiques de Bordeaux, Université Bordeaux-1, 351 Cours de la LibéraTion, 33405 Talence, France

E-mail address: andreeva@math.u-bordeaux.fr

Received 14/JUN/2007

Originally published in English 